\title{
Genomic lineages of Rhizobium etli revealed by the extent of nucleotide polymorphisms and low recombination
}

\author{
José L Acosta ${ }^{1 *}$, Luis E Eguiarte ${ }^{2}$, Rosa I Santamaría', Patricia Bustos', Pablo Vinuesa ${ }^{1}$, Esperanza Martínez-Romero ${ }^{1}$, \\ Guillermo Dávilaª and Víctor González ${ }^{1}$
}

\begin{abstract}
Background: Most of the DNA variations found in bacterial species are in the form of single nucleotide polymorphisms (SNPs), but there is some debate regarding how much of this variation comes from mutation versus recombination. The nitrogen-fixing symbiotic bacteria Rhizobium etli is highly variable in both genomic structure and gene content. However, no previous report has provided a detailed genomic analysis of this variation at nucleotide level or the role of recombination in generating diversity in this bacterium. Here, we compared draft genomic sequences versus complete genomic sequences to obtain reliable measures of genetic diversity and then estimated the role of recombination in the generation of genomic diversity among Rhizobium etli.
\end{abstract}

Results: We identified high levels of DNA polymorphism in R. etli, and found that there was an average divergence of $4 \%$ to $6 \%$ among the tested strain pairs. DNA recombination events were estimated to affect $3 \%$ to $10 \%$ of the genomic sample analyzed. In most instances, the nucleotide diversity $(\pi)$ was greater in DNA segments with recombinant events than in non-recombinant segments. However, this degree of recombination was not sufficiently large to disrupt the congruence of the phylogenetic trees, and further evaluation of recombination in strains quartets indicated that the recombination levels in this species are proportionally low.

Conclusion: Our data suggest that $R$. etli is a species composed of separated lineages with low homologous recombination among the strains. Horizontal gene transfer, particularly via the symbiotic plasmid characteristic of this species, seems to play an important role in diversity but the lineages maintain their evolutionary cohesiveness.

\section{Background}

Bacterial species typically contain large amounts of genetic variation in the form of single nucleotide polymorphisms (SNPs), which originate by mutation and have dynamics that depend on the balance between natural selection and genetic drift [1,2]. There is some debate on whether or not most of these polymorphisms are selectively neutral at the molecular level [3]. Species have been genetically defined through the analysis of DNA variation using comparative techniques such as hybridization, the sequencing of gene markers, and (more recently) complete genome sequences [4,5]. It has

\footnotetext{
* Correspondence: jlacosta@ccg.unam.mx

'Centro de Ciencias Genómicas, Universidad Nacional Autónoma de México, Av. Universidad N/C Col. Chamilpa, Apdo. Postal 565-A, Cuernavaca 62210, México

Full list of author information is available at the end of the article
}

been proposed that similarity values greater than $70 \%$ obtained in DNA-DNA hybridization experiments are sufficient to define a coherent group of organisms as belonging to the same species [6]. These estimates are very rough, subject to experimental variation, and they only indirectly measure similarity (i.e. via hybridization efficiency) [7]. A comparative analysis of complete genomes minimizes most of these limitations. Several measures of genomic relatedness, such as the Average Nucleotide Identity (ANI) and the Maximal Unique Matches (MUM) have been proposed for such analyses $[8,9]$. Both ANI and MUM are based on pairwise nucleotide comparisons of complete genomes, and several reports have shown good correlations between the results from these analyses and other measures of genetic relatedness, such as those based on Multilocus Sequencing Typing (MLST), 16S sequencing, and gene 
content [10]. However, these comparative methods rely on the availability of complete genome sequences and are affected by the quality of the DNA sequencing data, which in the case of draft genomes might not be optimal [10]. The latter issue has not been thoroughly addressed in past studies. One exception was the comparisons made by Richter and Roselló-Mora [10], who suggested that low genome sequence coverage can be sufficient for inferring DNA similarity values comparable to ANI obtained with complete genomes.

Bacterial species have mechanisms for gene exchange (transformation, conjugation and transduction), and genetic recombination is believed to play a prominent role in diversifying species by distributing variation and generating new allele combinations [11]. Horizontal gene transfer is an important source of genomic variation within and between species [12-16], and homologous recombination frequently results in the exchange of small genomic regions between members of the same or closely related species [17]. The estimated rates of homologous recombination vary widely among bacteria; in some instances, recombination seems to have contributed to species diversification to a greater extent than even point mutations, whereas in other species homologous recombination appears to be rare [18].

Recombination has typically been assessed by molecular techniques such as Multilocus Enzyme Electrophoresis (MLEE), Amplified Fragment Length Polymorphism (AFLP), or Multi Locus Sequence Typing MLST [19-21]. These methods primarily measure linkage disequilibrium (LD), and are based on the degree of allele association at different housekeeping loci. For example, E. coli strains show strong LD, reflecting infrequent genetic mixing within local populations [22]. More recently, the availability of complete genomic sequences has allowed recombination to be assessed more accurately [23]. Interestingly, genomic sequencing combined with analyses of population genetics have shown that the recombination rates within $E$. coli are higher than the mutation rates, but not to the extent that the phylogenetic signal is distorted [24]. Despite frequent recombination between strains, therefore, the genes seem to coexist in an organized genome, resulting in a chromosomal plasticity that accelerates the adaptation of E. coli to various environments.

In this work, we studied the intraspecific variability and recombination in Rhizobium etli, a soil bacterium that associates with bean roots to fix nitrogen. Previous studies have noted that this species has a variable gene content and high genomic divergence [24], as well as a low rate of recombination (in housekeeping genes) among isolates from the same geographical site $[22,25,26]$. However, in isolates (from the same geographical site) of Sinorhizobium medicae, it was found that frequency of recombination was higher in plasmids and megaplasmids, as compared to the chromosome [27]. The first purpose of this work was to perform a detailed genomic analysis of the nucleotide variation in this species. Accordingly, we used stringent methods to identify SNPs from a set of complete and draft genomes of $R$. etli, assessed the value of draft genomes and low coverage data when seeking to obtain global measures of genetic relatedness, and then examined the nucleotide differences among various strains of $R$. etli. The second purpose was to assess the role of recombination in generating genomic diversity in $R$. etli. Our results confirm and extend the previous estimations on the genomic diversity of $R$. etli, and indicate that recombination might play only a minor role in generating such diversity. Therefore, we conclude that the species $R$. etli is composed of separate genomic lineages that share a low rate of recombination but have a common symbiotic phenotype.

\section{Results}

\section{Nucleotide variation assessment in complete and draft} genomes

Since accurate SNP identification relies largely on the quality of the sequence data, the use of draft genome sequences could potentially introduce errors into the variation estimates. Therefore, stringent parameters (see Methods) were used to identify high-quality SNPs in a set of two complete $R$. etli genomes, CFN42 and CIAT652, isolated from México and Costa Rica respectively, and six draft genome sequences from strains isolated in different places of the world: BRASIL5 (Brazil), CIAT894 (Colombia), GR56, IE4771 (México), KIM5 (USA), and 8C-3 and GR56 (Spain) [24]. All the Sanger reads were collected from the draft genomes (about 13,000 reads of 1000 nucleotides in length per genome on average) were aligned against the predicted ORFs of the CFN42 or CIAT652 genomes, and the alignments were evaluated using Polybayes (additional file 1 Figure S1), which determined the probability that a nucleotide site was polymorphic, based on the Phred quality of the read. A Phred value of Q20 and a probability greater than 0.90 are generally considered acceptable for the detection of SNPs [28]. Most of the SNPs in our data set had probability scores $>0.975$, indicating that more than 100,000 SNPs per genome had Phred qualities over Q45 (additional file 1 Figure S1). To avoid the possible inclusion of false positives (in average 27,000 SNPs by each strain), we used only SNPs with a minimum Phred score of Q45 and the highest Bayesian probabilities (> 0.99) throughout this work [29].

Additional errors in SNP determination might arise from poorly aligned regions. Since $R$. etli genomes have a high proportion of paralogous sequences $[24,30]$, a 
stringent identification of orthologous segments of genes was performed. We aligned the contigs of each draft genome sequence against the ORFs from the complete genomes of either CFN42 or CIAT652, using both ungapped and gapped alignments, along with the reciprocal best hit criteria. We considered DNA gene segments as being orthologous to the reference sequence if they had nucleotide identities higher than $85 \%$ and coverage higher than $60 \%$ of the reference gene. Various numbers of orthologous segments were identified from the draft genomes, covering about $40 \%$ of the total gene contents of the reference strains. The total amount of data collected by this procedure is about 2 to $2.5 \mathrm{Mb}$ per draft genome (additional file 1 Table S1).

To determine the robustness of the above-described procedure, we simulated a draft assembly by using Sanger read samples of the complete genomes of different E. coli strains at low coverage $(1 \mathrm{x})$ (see Methods). The contigs of the simulated assembly were aligned with the genome of $E$. coli $\mathrm{K} 12$, and SNPs were detected as described above. On average, the obtained nucleotide variation ranged from about $1 \%$ to $2 \%$ (SNPs/alignment length) (Figure 1). There was no significant difference (p-value lower at 0.05 , according to Mann-Whitney and Kolmogorov-Smirnov tests obtained from Predictive Analytics Software PASW Statistics 18 (SPSS Inc.,

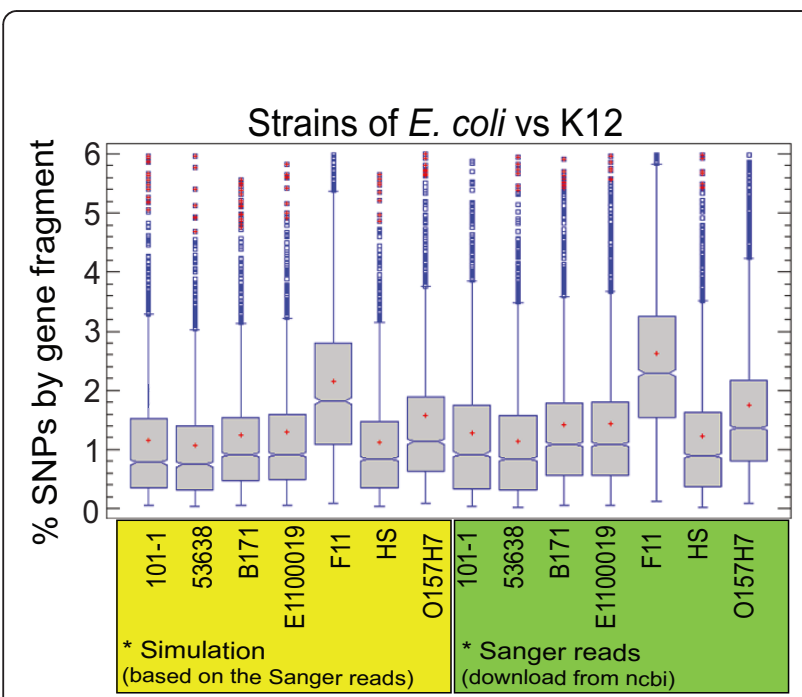

Figure 1 SNP assessment on lower coverage. Paired comparisons between E. coli strains and K12 strain as reference genome. We used two set of $E$. coli strains: Complete genomes obtained from Sanger reads (green box) and Sanger reads simulated from complete genomes with coverage approximate of $1 \mathrm{X}$ (yellow box). For each alignment, we determined the percentage of SNPs by gene fragment (SNP number/length of contig; $Y$ axis) by our methodology. Boxes inside the graphic include the median values (middle line) and the first and third quartiles (lower and upper lines) of the distribution. Abscissa: E. coli strains.
Chicago, IL)) when we compared the results obtained at $1 \times$ coverage versus those obtained with the complete genome assembled at about $10 \times$ coverage, indicating that $1 \times$ coverage of the genome sequence could be considered a robust proxy of full variation at the genomic level in this species.

\section{SNP frequencies among the $R$. etli strains}

We quantified the SNPs in $R$. etli by computing the pairwise nucleotide differences between individual draft genomes versus the complete genomes of strains CFN42 or CIAT652. More SNPs were found in comparisons made versus the CFN42 genome (Figure 2, gray boxes) than the CIAT652 genome (Figure 2, blue boxes). For example, the BRASIL5 strain had a median of 5\% SNPs per aligned fragment when compared with CFN42 but only $2 \%$ compared to CIAT652, indicating that BRASIL5 is more closely related to CIAT652 than CFN42. Similarly, variance was higher when BRASIL5 was compared with CFN42 rather than CIAT652 (Figure 2). A very similar pattern was found for strain $8 \mathrm{C}-3$. The other strains showed similar levels of variation, on the order of $6 \%$ (CFN42) and 4\% (CIAT652), with the latter comparison always showing a lower variance. Comparison between the complete genomes of CFN42 and CIAT652 (Figure 2, red box) result in a median variation of $9 \%$, that is high but still lower than the comparisons between CFN42 and R. leguminosarum bv viciae 3841 (Figure 2 green box). Moreover, when we compared $R$. leguminosarum bv. viciae 3841 with all of the $R$. etli strains (complete and draft genomes) (additional file 1

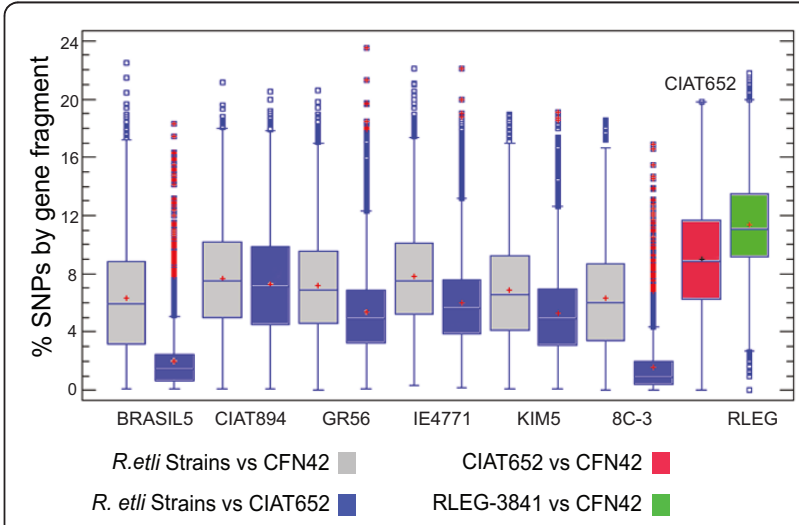

Figure 2 Paired comparisons between $R$. etli strains. We performed four paired comparisons used our methodology: draft genomes of $R$. etli against CFN42 (gray boxes), draft genomes of $R$. etli against CIAT652 (blue boxes), CIAT652 against CFN42 (red boxes) and finally $R$. leguminosarum bv viciae 3841 against CFN42 (green boxes). For all comparisons the $\mathrm{Y}$ axis is the percentage of SNPs by gene fragment (SNP number/length of contig). Boxes inside the graphic include the median values (middle line) and the first and third quartiles (lower and upper lines) of the distribution. Abscissa: R. etli and R. leguminosarum bv viciae 3841 strains. 
Figure S2), the greatest difference in SNP percentage (median 11\%) was seen in the comparison with strain CFN42 (Figure 2 green boxes, and discussion section).

\section{Average nucleotide variation}

We sought to obtain a single measure of the nucleotide variation across the whole set of genomes. To this end, we averaged the medians of the SNP distributions for each alignment (i.e., the number of SNPs/alignment length of each draft genome with respect to CFN42 or CIAT652) and generated average confidence interval (obtained and adjusted by distribution of genes size medians) using Predictive Analytics Software PASW Statistics 18 (SPSS Inc., Chicago, IL). This statistical test of proportions compares the observed proportions of an event (here, SNPs) in k samples (here, strains), uses a chi-squared test to seek significant differences among the proportions, and subsequently adjusts the confidence intervals for each sample. The generated measure, herein called the average nucleotide variation (ANV), might represent the species-level variation. We obtained ANV values of $4 \%$ and $6 \%$ when we compared all the analyzed strains against CIAT652 and CFN42, respectively (Figure 3). Although the largest numbers of SNPs were found in comparisons with the CFN42 genome, all strains were similarly divergent according to the $95 \%$ confidence intervals with respect to the median (blue lines in Figure 3). This observation indicates that CFN42 is almost equally divergent with respect to all other strains. Comparisons with the CIAT652 genome showed that strains BRASIL5 and $8 \mathrm{C}$-3 were closer to this strain than to CFN42. Moreover, the CIAT894 strain yielded the highest number of SNPs, causing its average SNP proportion to fall outside the average confidence interval (red lines in Figure 3). Strains CIAT894 and IE4771 showed greater divergences than the rest of the strains, regardless of the reference strain (CFN42 or CIAT652) used in the comparison.

\section{Nucleotide variation profiles in homologous genomic segments from different $R$. etli strains}

To explore how SNPs are distributed in the $R$. etli genomes, we first identified orthologous segments for which we had sequence information in all eight studied strains (Figure 4). A total of 240 segments with a median size of $275 \mathrm{bp}$ were common to all strains, and spanned a total of about $71,630 \mathrm{bp}$ that represent about $1 \%$ of the genome length. These sequences mapped mainly to the chromosomes of CFN42 and CIAT652 (92\%), with a lower proportion (8\%) distributing to plasmids. We generated a concatenated alignment of these shared segments according to the gene order found in the CFN42 genome, and then inferred a consensus sequence and computed the number of nucleotide differences across

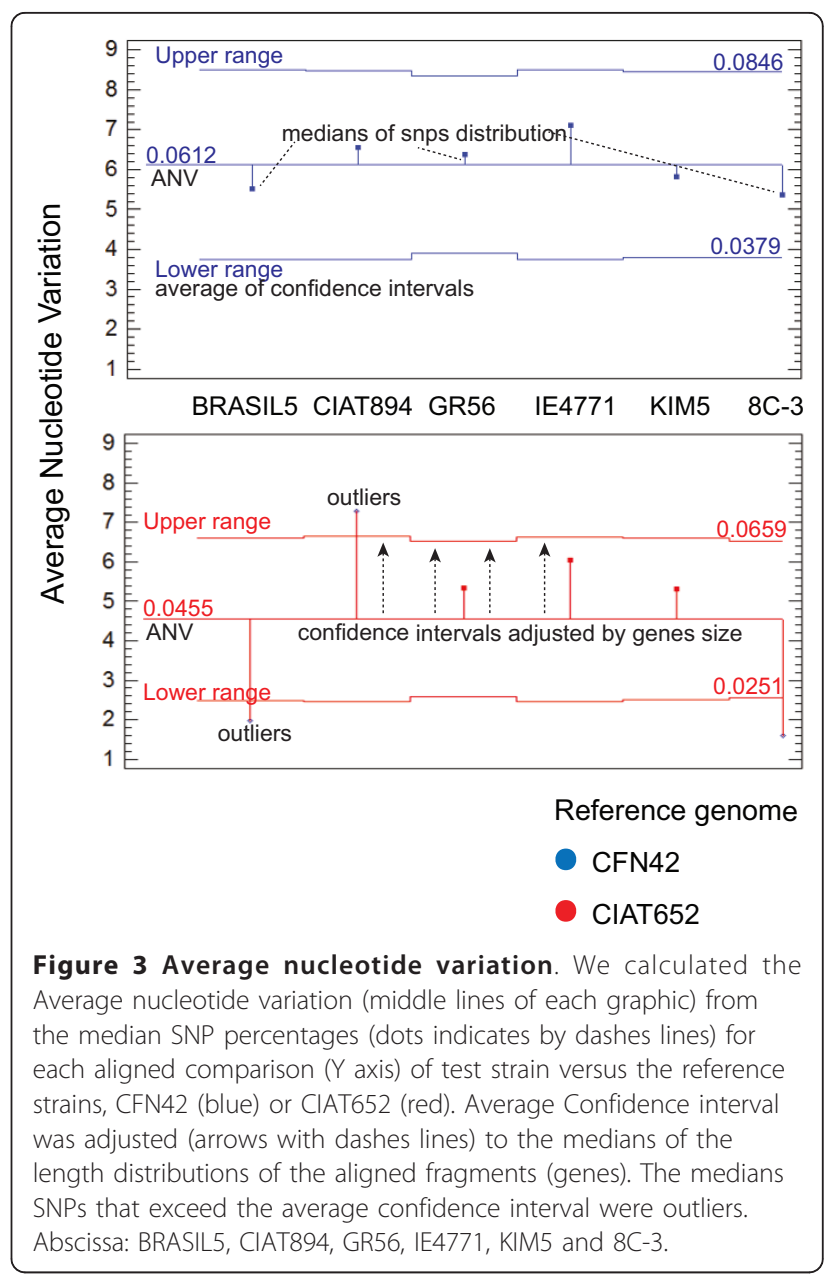

windows of $250 \mathrm{bp}$. Using this procedure, we detected the patterns of shared and unique (singleton) SNPs particular to each strain. As shown in Figure 4, we were able to distinguish two classes of shared SNPs: biallelic SNPs (Figure 4 gray smoothed areas), which showed only one nucleotide difference with respect to the consensus; and polyallelic (Figure 4, white bars), which showed multiple differences at the same nucleotide site with respect to the consensus. Some of these SNP patterns were shared in some strains but not others. For example, as shown in Figure 4, pattern A was shared by strains CIAT652, CIAT894 and 8C-3, whereas pattern B was found in strains GR56, IE4771 and Kim5. Further shared patterns were identified through a careful inspection of the plot. In addition, a large number of polymorphisms were not shared, but instead appeared to be strain-specific variants. Interestingly, strain CFN42 was found to have the greatest number of differences with respect to the consensus (Figure 4, black bars). Even thought this approach is limited by the amount of common segments among the eight strains, we were able to cover $3.7 \%$ (223) of the total gene content $(5,963)$ of the 


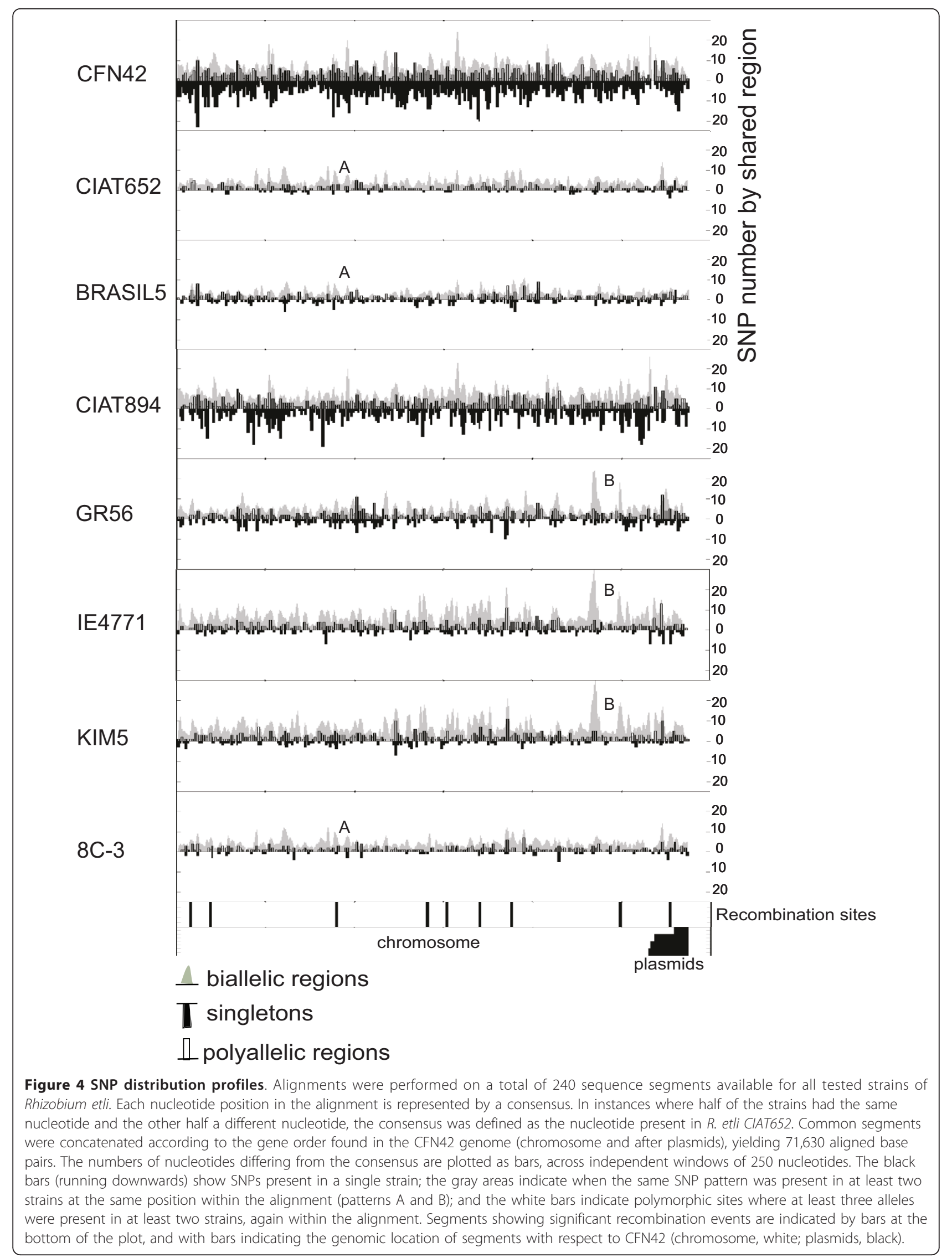


CFN42 reference strain that include the main COG categories and subcategories (see Methods). For instance, metabolism (transport and metabolism of sugar, amino acids, and carbohydrates); cellular processes and signaling (envelope biogenesis, signal transduction); information storage and processing (transcription, replication, and recombination); and poorly characterized proteins (function unknown). A detailed annotation of the gene segments can be seen in additional file 2 Table S1.

\section{Phylogenetic congruence}

Since recombination can distort phylogenetic trees such a way that no two individual trees are topologically equivalent, we decided to perform phylogenetic reconstructions using a) a neighbor-joining network [31]; and b) a comparison of a consensus tree with individual trees constructed using the 187 segments common to the eight studied $R$. etli genomes and $R$. leguminosarum $b v$ viciae 3841 (RLEG). The consensus trees obtained from the concatenated alignments had identical topologies when constructed by maximum likelihood, Bayesian, and neighbor joining network methods (see Methods). Only the tree based on neighbor joining network is shown in Figure 5. This tree was found to contain six internal branches (denoted by split numbers). There are two main clusters in the tree, separated by branches 2 and 3 that group the most closely related strains: one containing KIM5, IE4771, and GR56 (branch 2) and another grouping BRASIL5, 8C3, and CIAT652 (branch 3). These branches are internal in

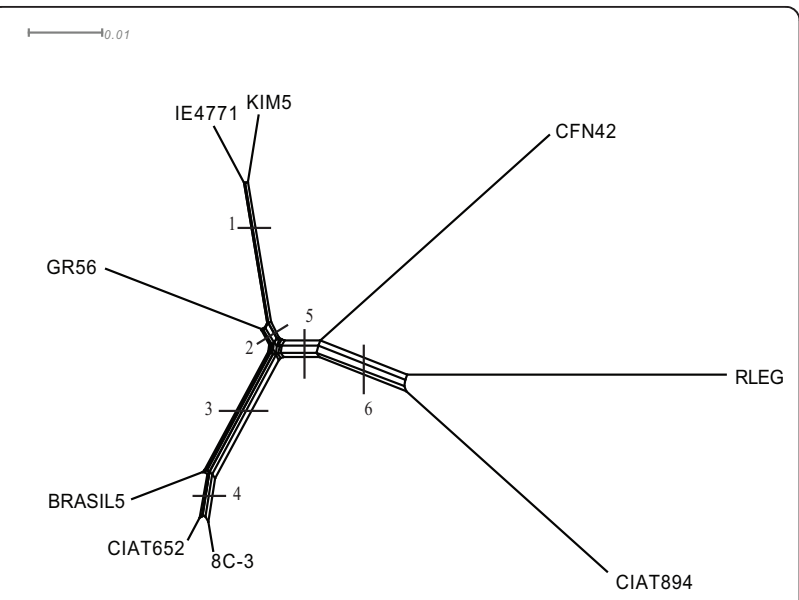

Figure $\mathbf{5}$ Genetic relatedness. Network joining network phylogeny inferred from 186 concatenated regions shared among strains of Rhizobium etli and Rhizobium leguminosarum bv viciae 3841 (see Methods). The tree is unrooted and has six internal branches, indicated by split numbers on each internal branch. The scale bar denotes the expected number of nucleotide substitutions per site. relation to branch 5, which separates CFN42, CIAT894, and RLEG that are the strains with the longest branches (greatest number of nucleotide substitution per site). A few inconsistencies were found among the topologies recovered from reconstructions based on individual gene segments (187), as compared to the topology of the consensus tree (not shown). These alternative topologies are mainly due to the position of CIAT894 and RLEG, whereas the splits 2,3 , and 5 where consistently recovered. Thirty out of 187 trees supported the placement of RLEG as the most distant strain, 39 trees supported placement of CIAT894 as the external strain, whereas the most frequent topology shows that these strains are equally distant to the rest of strains (Figure 5). These alternative topologies could be the result of shared ancestral polymorphisms, as suggested by the long branches coupled with low frequency of recombination. Altogether, the phylogenetic reconstructions suggested that the levels of recombination were insufficient to erase the phylogenetic signal, thus allowing for the identification of the most probable strain tree. Consistent with this conclusion, only nine $(3.75 \%)$ of the 223 gene segments common among the eight $R$. etli strains (Figure 4) showed at least one recombination event.

\section{Extent of recombination}

To evaluate the extent of the probable recombination events among strains of $R$. etli, we performed a recombination analysis in orthologous quartets (see Methods). We aligned the shared gene segments from each draft genome with the corresponding segments of the ORFs from CFN42, CIAT652, and the R. leguminosarum bv viciae 3841 complete genomes, yielding six different groups of quartets (one group for each incomplete genome; Figure 6). The proportion of aligned segments varied across the six groups of quartets, from $\sim 2,781$ segments in the group containing BRASIL5, to $\sim 3,672$ in the group containing CIAT894. The segments ranged from 200 to $4651 \mathrm{bp}$ in length and covering approximately $50 \%$ of the genome (additional file 1 Table S2). For each group of quartets, we performed four different recombination tests (see Methods), and determined the number of recombination events (only those that were detected by at least two methods) for each quartet (describe above) (Figure 6). The lowest proportions of recombination events were detected for the quartets containing strains BRASIL5 and 8C-3, which showed $4.42 \%$ (123 out 2781 ) and $3.57 \%$ (102 out 2854 ) recombination events, respectively. The other groups showed approximately twice as many recombination events, with frequencies ranging from $8.67 \%$ (KIM5 quartets) to $10.86 \%$ (GR56). In addition, for each group of recombinant quartets, we determined the number of events of recombination between pairs of strains (Figure 6). In 




general, recombination events were more frequently predicted between $R$. etli strains pairs than between any given $R$. etli strain and $R$. leguminosarum bv viciae 3841 (Figure 6). For instance, in the group of quartets containing BRASIL5, the percentage of recombinant segments is about 7\% in CFN42-RLEG, 5\% in BRASIL5RLEG, and 20\% in CIAT652-RLEG pairs, whereas recombinant segments were detected more frequently between pairs of $R$. etli strains: 18\% (CFN42-BRASIL 5), 25\% (CFN42-CIAT652), and 25\% (CFN42-CIAT652). The same pattern was seen for the other five groups of quartets. This effect is because homologous recombination depends on a high nucleotide identify, and greater divergence is associated with less homologous recombination [32]. Therefore, recombination might be more frequent between strains (populations) that are closely related. Indeed, we observed the same recombination events in different groups of quartets (of different strains), as indicated by a presence/absence matrix. In general, the number of common recombination events (small number of events) was related to the phylogenetic proximity of the strains, for instance BRASIL5 and 8C-3 share the most recombination events in common (data not shown).

To explore whether the recombination is particularly acting on some classes of genes, we assigned the 
recombinant segments to COGs (see Methods), as shown additional file 1 Figure S3. All the functional classes annotated in the CFN42 genome are present in the draft genomes but they are represented unevenly in the recombinant segments. For instance, the categories: amino acid transport and metabolism, carbohydrate transport and metabolism, energy production and conversion, lipid transport and metabolism, general function prediction only and function unknown appear overrepresented among the recombinant segments. In counterpart, some other categories like transcription and signal transduction mechanisms are in lower frequency among the recombinant segments than in CFN42. Even though we performed a chi-square and Range tests [33] to assess the significance of these differences, the incomplete nature of draft genomes does not allow to conclude about some bias toward recombination in certain classes of genes.

\section{Genetic diversity}

Together the above-described data suggest that recombination may not be a major driver of genomic diversification in $R$. etli, but rather might have relatively limited effects. To directly examine this point, we estimated the mean nucleotide diversity per nucleotide site $(\pi)$ for the recombinant and non-recombinant gene segments of each strain (Figure 7a). In general, recombinant segments showed higher $\pi$ values than non-recombinant segments. These differences were significant only for strains CIAT894, GR56, IE4771 and KIM5 (Student's $t$ test, $\mathrm{p}<0.001$ ), but the combined data for the $\pi$ values of the 240 recombinant and non-recombinant gene segments common to the eight strains showed the lowest $\pi$ values ( 0.06 on average). Although there was no significant difference between recombinant (red circles) and non-recombinant segments (blue circles) with regard to the regions common to all eight strains (Figure 7b), most of the recombinant segments had higher-thanaverage $\pi$ values and generally showed the highest transition/transversion ratios (indicated by the size of the circles in Figure 7b). Since the probability of transitions is higher than transversions [34], high ratios of transition/transversion suggest that they were under strong purifying selection, because transitions at the third 'wobble' position are more likely to be synonymous than transversions [35].

\section{Discussion}

In the present work, we used a genomic approach to detect and measure variation in the form of SNPs, and to analyze the contribution of recombination to the genomic diversification of $R$. etli strains. Our results demonstrated that draft genomic sequences samples representing $\sim 1 \times$ of the genome can be used to measure

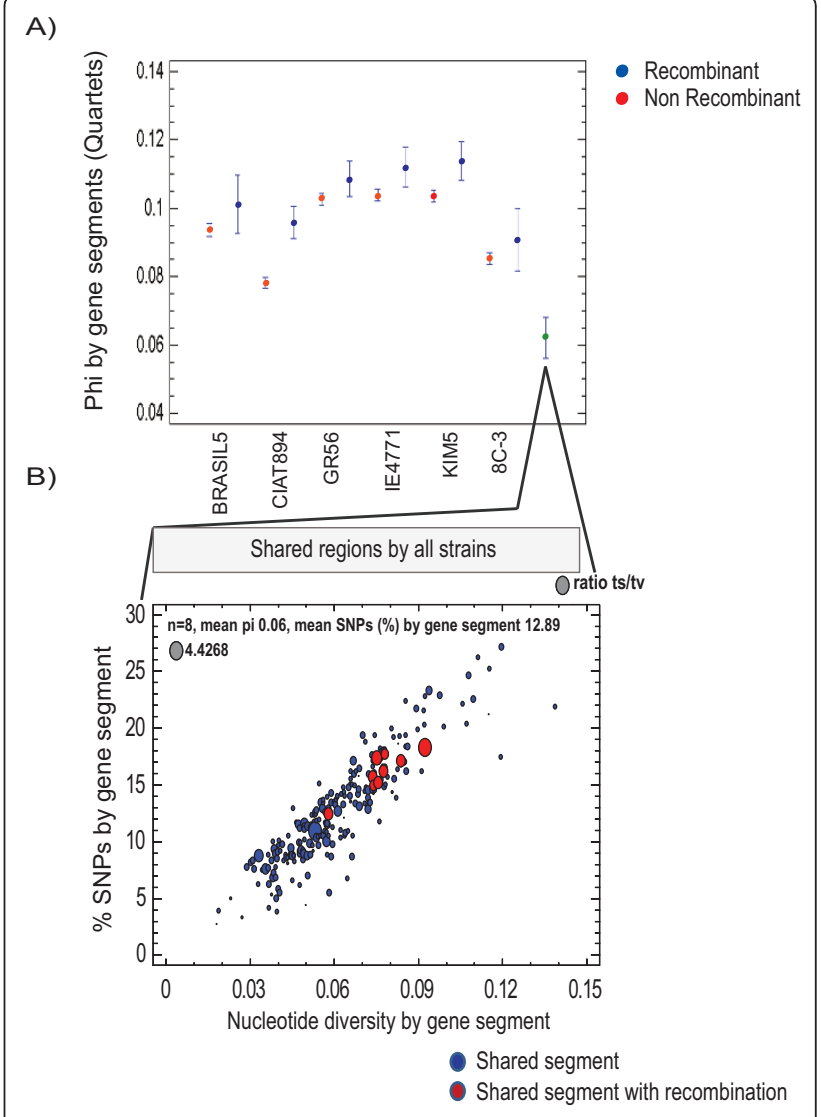

Figure 7 Genetic diversity in recombinant segments. A) For each homologous segment (quartet; regardless of evidence of recombination), we calculated the nucleotide diversity phi ( $Y$ axis; see Methods). The dots indicate the distribution means and the bars represent the $95 \%$ confidence intervals. Blue and red dots indicate recombinant and non-recombinant segments, respectively. Moreover, we determined the nucleotide diversity of the sequence regions shared across all of the tested strains of $R$. etli (green dot). Abscissa: BRASIL5, CIAT894, GR56, IE4771, KIM5, 8C-3 and shared regions. B) Magnification of the results from the 240 common sequence segments shared by all tested strains. The average percentage of SNPs was $12.89 \%$ per segment. The sphere sizes indicate the proportions of ts/tv for both recombinant segments (red circles) and non-recombinant segments (blue circles). The $Y$ axis denotes the SNP percentage by gene segment, while the $X$ axis shows the nucleotide diversity.

variation at the whole-genome level in this species. In $R$. etli we found a great amount of variation (more than 161,998 SNPs) when any draft genome was compared to the complete genomes of CFN42 and CIAT652. To assess the reliability of this method for identifying SNPs, we quantified the SNPs in E. coli genomes at $1 \times$ and in complete genomes assembled at about $10 \times$ coverage. We found the same variation level using either draft or complete E. coli genomes, indicating that draft genomes produced estimations of DNA variability comparable to those generated using complete genomes even at only 
$1 \times$ coverage. Richter and Roselló-Mora [10] previously reported on the use of partial sequences representing about $20 \%$ of the genomes of several bacterial species to infer reliable values of DNA divergence between strains. The authors of the prior paper showed that ANI values obtained with these samples correlated well with the $\mathrm{DDH}$ values, indicating that draft genome sequences are an acceptable data source. At present, the rapid improvement of DNA sequencing technology is allowing researchers to use multiplex sequencing to simultaneously process an increasing number of genomic sequences. These experiments will produce additional draft genome sequences of different qualities, and the approach proposed herein should prove useful for their early analysis.

We identified a higher proportion of SNPs in $R$. etli strains than in E. coli strains, and the differences between the various $R$. etli strains and Rhizobium leguminosarum bv viciae 3841 ranged from $7 \%$ to $11 \%$ (median; additional file 1 Figure S2), with the latter figure corresponding to the CFN42 comparison. $R$. etli and $R$. leguminosarum are different species according to $16 \mathrm{~S}$ comparison; however, they share a common genomic core and are distinguished by variable accessory components (e.g., plasmids) [24,36,37]. Therefore, an ANV range of $7-11 \%$ might be a good indicator of speciation within Rhizobium. Despite of the variability in ANV among the tested strains of $R$. etli (about 4-6\%), none had ANV values comparable to those obtained with respect to $R$. leguminosarum. The levels of ANV were higher for comparisons using CFN42 than those done with CIAT652. For taxonomic purposes, CFN42 is the type strain of $R$. etli [38]. In the present analysis, however, we found that CFN42 was the most differentiated of the studied samples, had the highest proportion of unique SNPs, and clustered as a divergent independent branch when the strain phylogeny was explored. We recently re-sequenced strain CFN42 using Solexa-Illumina technology and compared it with the former complete genome sequence. Very few indels and SNPs (less than 20 SNPs) and no rearrangements were found. Therefore, very small variation can be expected from an in vitro lifestyle. In contrast, most of the strains analyzed were more closely related to CIAT652 than to CFN42. A prior study noted that CIAT652 and CFN42 have a low ANI value (90.44\%) [10] and suggested that CIAT652 is improperly classified as $R$. etli. We previously showed that CFN42 and CIAT652 share a very conserved symbiotic plasmid, but have high divergence throughout the rest of their genomes [24]. Given that all isolates of $R$. etli have been recovered from nitrogen-fixing bean nodules, this characteristic would be expected to dominate the classification criteria. The genomic divergence described herein is thus consistent with a model in which the species $R$. etli is composed of divergent genomic lineages that share the symbiotic phenotype conferred by the symbiotic plasmid [24], which is called a common symbiovar [39]. Indeed, our analysis suggests that in some instances, the use of type strains could lead to misleading taxonomic classifications, especially when gene transfer mechanisms are active. $R$. etli is known to have mobile elements such as conjugative plasmids, insertion sequences and bacteriophages [40-42]. Therefore, gene flow and recombination among strains of $R$. etli might be important to the production of genomic diversity, as reflected in its pangenomic structure [24]. However, no prior study has assessed the role of homologous recombination in promoting the genomic diversity of $R$. etli. Earlier works using MLEE or MLST concluded that $R$. etli populations are essentially clonal, with low recombination even in sympatric populations [22,25,26,43]. More recently, Flores et al. [44] showed that despite the high conservation of the symbiotic plasmid pSym sequences from a collection of different strains of $R$. etli, some regions shared identical SNP distribution profiles. This observation was interpreted as evidence of homologous recombination. Here, we obtained similar findings for a set of common genomic DNA segments, mainly chromosomal in origin, belonging to eight strains of $R$. etli. Quantification of probable recombination events and the extrapolation of our findings to the whole genome suggested that a minimum of 260 recombination events had occurred in the genome of each strain. Strains CFN42 and CIAT894 were the more variable in terms of SNPs, and the latter also showed the most evidence for recombinant events in our quartet analysis (within the orthologous segments). Even though there were some discrepancies within the clades of the various phylogenetic trees we generated, most of the trees were congruent with the consensus tree. Moreover, although the estimated recombination was correlated with genetic diversity (Figure $7 \mathrm{a})$, it was low overall (3-10\%). In comparison, the whole-genome recombination estimates reported for Rickettsia and Streptococcus were on the order of 18$37 \%$ and $28 \%$, respectively $[45,46]$. These data suggest that only a minor fraction of the $R$. etli genome has undergone recombination, which thus accounts for only a low proportion of the polymorphism in this species.

In bacteria, the frequency of RecA-mediated homologous recombination depends on the level DNA identity, and small DNA fragments are often introduced into the cell via conjugation, transformation or transduction. Consequently, only a fraction of the genome might be targeted by recombination [47]. Several other factors might account for the low recombination frequency detected in the isolate of $R$. etli studied here. Among them, the ample degree of divergence among the studied 
$R$. etli strains, their distant geographical origins (USA, México, Costa Rica, Colombia, Brazil, and Spain) [24], and the small number of sampled strains. Recently, Bailly et al., reported a population genomics analysis of sympatric strains of Sinorhizobium medicae [27]. They found very low levels of polymorphism and recombination in the chromosome in comparison with the megaplasmids. Future studies using our methodology on $R$. etli isolates from single sites could be used to improve our understanding of how recombination impacts the diversification of this species.

\section{Conclusion}

In summary, our results and the previous reports on $R$. etli support a model in which the species is composed of evolutionarily independent lineages that share a symbiotic phenotype but have low levels of recombination among the various lineages. However, although genetic barriers imposed by divergence or other barriers such as geographical distance might preclude homologous recombination among the strains, gene flow (e.g., by plasmids and chromosomal islands) is an ongoing process that shapes the genomic and pangenomic structures of $R$. etli.

\section{Methods}

\section{Genomes used}

Complete genome sequences were downloaded from GenBank as follows: for $R$. etli CFN42: chromosome [GenBank:NC_007761], and plasmids pCFN42a [GenBank: NC_007762], pCFN42b [GenBank:NC_007763], pCFN4c [GenBank:NC_007764], pCFN42d [GenBank:NC_004041], pCFN42e [GenBank:NC_007765], and pCFN42f [GenBank:NC_007766]; for $R$. etli CIAT652: chromosome [GenBank:NC010994], and plasmids pCIAT652a [GenBank:NC010998], pCIAT652b [GenBank:NC010996], and pCIAT652c [GenBank:NC010994]; and for R. leguminosarum 3841: chromosome [GenBank:NC_008380], and plasmids pRL7 [GenBank:NC_008382], pRL8 [GenBank: NC_008383], pRL9 [GenBank:NC_008379], pRL10 [GenBank:NC_008381], pRL11 [GenBank:NC_008384], and pRL12 [GenBank:NC_008378]. We also used reads and contigs from the draft genomes of $R$. etli strains $8 \mathrm{C}-3$ [GenBank:NZ_ABRA00000000], BRASIL5 [GenBank: NZ_ABQZ00000000], CIAT894 [GenBank: NZ_ABRD0000000], GR56 [GenBank: NZ_AABRD0000000], IE4771 [GenBank: NZ_ABRD00000000], and KIM5 [GenBank: NZ_ABQY0000000].

\section{Determination of SNPs and pairwise nucleotide differences}

Paired alignments between the draft genomes (contigs) and the ORFs from the genomes of CFN42 or CIAT652 were performed using the Dds2 program [48], which produces ungapped alignments of fragments having similarities greater than $80 \%$. Each duplicated paired alignment (i.e., segments for which paralogous existed in the reference genome) was filtered using the reciprocal best hits option of the Fil program [48] under the following parameter set: coverage $>60 \%$ with respect to a reference gene and a percentage differential score cutoff $<10 \%$. When two alignments had the same coverage, we selected the alignment with the higher score. Once the results were filtered, we created a gapped alignment using the Gap22 program [48] on segments for which the identity was greater than $85 \%$. Both sequences were extracted using an ad hoc Perl script (homemade) formed for each paired alignment. To avoid frameshifts, we realigned each pair using cross-match [49] with the following parameters: discrep_lists masklevel, 0; tags gap_init, 3; gap_ext, 2; ins_gap_ext, 2; del_gap_ext, 2; minmatch, 14; maxmatch, 14; max_group_size, 20; minscore, 30; bandwidth, 14; and indexwordsize, 10. Finally, for each alignment, we determined the probability that a site was polymorphic using the Polybayes program [50], with the probability set at greater than 0.99 and a minimum Phred of Q45 [51,52].

\section{Assessment of methodological accuracy at low coverage}

To determine if differences in coverage among the studied strains affected the reliability of the variability estimations, we took readings representing $\sim 1 \times$ sequence coverages of seven $E$. coli genomes and complete-genome readings (about $10 \times$ ) of the same genomes from GenBank (ftp://ftp.ncbi.nih.gov/genomes/) and assembled these readings using the Celera assembler [53]. The above-described analysis was applied to both the $1 \times$ and $10 \times$ coverage datasets, and the results were compared using the Mann-Whitney and KolmogorovSmirnov tests [33]. The utilized E. coli draft genomes were: 101-1 [GenBank:NZ_AAMK00000000], 53638 [GenBank:NZ_AAKB00000000], B171 [GenBank: NZ_AAJX0000000], E1100019 [GenBank: NZ_AAJW0000000], F11 [GenBank:NZ_AAJU0000000], HS [GenBank:NC_009800], and O157_H7_ec4024 [GenBank:NZ_ABJT0000000]. The complete genome sequence of E. coli K12 [GenBank:NC_000913] was used as the reference.

\section{Determination of triplets (homologous segments)}

For the comparisons between all ORFs of the reference genomes (both CFN42 and CIAT652 were used throughout the work) and each incomplete genome (the contigs), we obtained the coordinates of all homologous segments (triplets) using the Mauve program [54]. Our analysis was standardized by aligning p42F (CFN42) against ( $R$. leguminosarum bv viciae 3841) pRL12 using 
the following parameters: backbone-size $=100$; $\max -$ backbone-gap $=50$; weight $=90$; island-size $=100$. These plasmids were chosen because they contain shared syntenic blocks [36]. Sequence extraction, realignment of each conserved segment (backbone) and SNP determination were all performed as described above (see determination of SNPs section).

\section{Determination of quartets (orthologous segments)}

To detect recombination events among DNA sequences, at least four sequences are required for the analysis [55]. Here, we first identified SNPs that distinguished each draft genome from the two reference genomes (CFN42 and CIAT652), and then determined the fragments that were shared between each draft genome and the ORFs from CFN42 and CIAT652, together with all replicons of $R$. leguminosarum 3841 (chosen because of its extensive synteny with CFN42) [36]. Sharing was determined using the Mauve program [54] (see determination of triplets section) and the shared fragments were realigned with the Muscle program (default parameters) [56]. To eliminate any large gaps within the alignments (rare in orthologous fragments), we used the Gblocks program under its default parameters [57].

\section{Detection of recombination}

A variety of methods for detection of recombination have been reported in the literature [58], but no one strategy performs optimally under all evolutionary scenarios [59]. Therefore, a reasonable approach is to employ multiple methods and consider recombination events predicted by at least two methods as being the most reliable. Here, we used this strategy and considered recombination events that were detected by at least two of the following four programs [46]:

A) Geneconv [60]: Using this program, we ran 100,000 simulations for each quartet with the following parameters chosen: Dumptab; Dumpjseq; Dumpfrag; Annotate; WideCol; ShowBlast; Indel_blocs; ShowBcPwKaPvals; SortGfragsBySeq; Show_maxmeansims; ShowUnal; Gscale = 1; ListPair; ListBest; Bcsims; Allouter; Numsim $=100000 / \mathrm{sp}$. This allowed us to detect possible genetic conversion events.

B) Pist [61]: With this program, we first identified the best-fit DNA substitution model for each shared fragment using the Akaike information criterion. We then used the best model to reconstruct the phylogeny using a maximum likelihood method (Phyml [62]) with 100 non-parametric bootstrap replicates. We next determined the invariant sites, alpha values, ts/tv ratios, base frequencies, and constant sites using the PAML program [63] and the GTR model. Finally, we ran Pist with the REV model and 10,000 permutations. Pist uses parsimony-informative sites to detect recombination events and is robust for highly divergent genes.

C) PhiPack [64]: We used the parameters of 10,000 permutations and a window size of $25 \mathrm{nt}$, and implemented the Pairwise Homoplasy Index, Maximum X2, and the Neighbor Similarity Score.

D) Hyphy program [65]: We used the routine GARD, which enables automated phylogenetic detection of recombination. We employed the GTR model and betagamma rate variation.

To determine if a recombinant gene was present in two different quartets or strains, we constructed a binary presence/absence matrix $(1 / 0)$ for each gene that was found in two or more strains. These profiles were hierarchically clustered using the Cluster program [66].

\section{Phylogenetic analysis}

Regions shared among all strains of $R$. etli and $R$. leguminosarum bv viciae 3841 were identified using the Mauve program [54], realigned by Muscle using the default parameters [56], and filtered for long gaps with Gblocks [57]. We then obtained the phylogeny of each region using a maximum likelihood approach employed by the Phyml program [62] (with 1,000 non-parametric bootstrap replicates) and the best nucleotide substitution model identified by the Akaike information criterion $[67,68]$. We used three methods to construct the phylogeny from the concatenated dataset, in order to determine the species tree. The first was the RAxML program (maximum likelihood) [69], in which we ran the GTR nucleotide substitution model and a GAMMA + P-Invar estimation of rate heterogeneity. This analysis yielded a Maximum Likelihood ML estimate of the alpha parameter and 1,000 distinct randomized Maximum Parsimony trees. The second program used was Phyml (maximum likelihood) [62], running 1,000 nonparametric replicates and the GTRG model. Finally, we employed the MrBayes program (Bayesian analysis) [70] running the Nucmodel 4by4 for DNA. The number of rate categories for the gamma distribution was set at four, with an allowance for a proportion of invariable sites. Because of the high computational burden, we performed two runs with four chains, for 500,000 generations in total. Trees were sampled every 500 generations, $25 \%$ of all samples were removed as reflecting burn-ins, and a consensus was obtained. Moreover, to assess differences in topology among the probable strain trees and individual gene trees, we used the Consel program [71], which calculated expected likelihood weighting and performed the Shimodaira-Hasegawa SH test [72]. Finally, a neighbor-net network was generated using the concatenated sequences and the Splits tree4 program [31]. 


\section{Nucleotide diversity and ts/tv ratios}

For each shared fragment (quartet), we determined the nucleotide diversity and segregating sites using $R$. leguminosarum 3841 as an outgroup and employing the libsequence library [73]. The transition/transversion ts/tv ratios were determined for each quartet by using the PAML program [63] and applying the best model of nucleotide substitution obtained from each orthologous segment (see determination of quartet).

\section{Functional assignment}

We used the COGs database [74] to undertake functional annotation across the four broad categories and sub categories to shared regions (all strains) as well as recombinant quartets. Quartets that had not been functionally assigned within the COG database were placed in the "Poorly Characterized" category. For assignation to a category, we used the reciprocal best hits technique with an E-value $<1 \times 10^{-7}$.

\section{Additional material}

Additional file 1: Strategy for Determining SNPs. The additional file (in .pdf format) includes text and figures delineating our process for determining SNPs (parameters, paired comparisons and SNP differences). Also include the distribution of functional classes (COGs) of recombinant quartets of each draft genome and your comparison against distribution of CFN42.

Additional file 2: Table of genes presented in $\mathbf{2 4 0}$ shared regions. The additional file (in .xls) includes the tables of genes and your features, as name, coordinates, gi, COGs and other.

\section{Acknowledgements}

We thank José Espiritu, Ismael L Hernández, and José L Fernández for their help with technical and computational resources, and Miguel A Cevallos for critical reading of the manuscript.

This work was supported by grants from CONACyT (CB131499 and U4633) and PAPIIT-UNAM (IN215908 and IN223005). JLA received a Ph. D. fellowship from CONACYT. We thank the Doctorate in Biomedical Sciences and specially to National Autonomous University of México.

LEE contributed during a sabbatical visit to the University of California Irvine $(\mathrm{UCI})$ in the laboratory of Brandon Gaut; this work was supported by UCMEXUS, CONACYT, and DGAPA, UNAM.

\section{Author details}

'Centro de Ciencias Genómicas, Universidad Nacional Autónoma de México, Av. Universidad N/C Col. Chamilpa, Apdo. Postal 565-A, Cuernavaca 62210, México. ${ }^{2}$ Departamento de Ecología Evolutiva, Instituto de Ecología, Universidad Nacional Autónoma de México, CU, AP 70-275 Coyoacán, 04510 México, DF, México.

\section{Authors' contributions}

JLA conceived, designed, and performed the experiments. JLA and VG analyzed the data and wrote the manuscript. RIS and PB were responsible for the genomic sequencing. PV, LEE and EM-R discussed the data. GD and VG contributed materials. VG edited the manuscript and is the Ph. D. thesis advisor of JLA. All authors read and approved the final manuscript.

Received: 1 June 2011 Accepted: 17 October 2011

Published: 17 October 2011
References

1. Kimura M, Takahata N: Selective constraint in protein polymorphism: study of the effectively neutral mutation model by using an improved pseudosampling method. Proc Natl Acad Sci USA 1983, 80(4):1048-1052.

2. Nei M: Selectionism and neutralism in molecular evolution. Mol Biol Evol 2005, 22(12):2318-2342.

3. Wagner A: Neutralism and selectionism: a network-based reconciliation Nat Rev Genet 2008, 9(12):965-974.

4. Konstantinidis KT, Ramette A, Tiedje JM: The bacterial species definition in the genomic era. Philos Trans R Soc Lond B Biol Sci 2006, 361(1475):1929-1940.

5. Rossello-Mora R, Amann R: The species concept for prokaryotes. FEMS Microbiol Rev 2001, 25(1):39-67.

6. Wayne LG, Brenner DJ, Colwell RR, Grimont PAD, Kandler O, Krichevsky MI Moore WEC, Murray RGE, Stackebrandt E, Starr MP, et al: Report of the ad hoc committee on reconciliation of approaches to bacterial systematics. Int J Syst Bacteriol 1987, 37:463-464.

7. Konstantinidis KT, Ramette A, Tiedje JM: The bacterial species definition in the genomic era. Philos Trans R Soc Lond B Biol Sci 2006, 361(1475):1929-1940.

8. Konstantinidis KT, Tiedje JM: Towards a genome-based taxonomy for prokaryotes. J Bacteriol 2005, 187(18):6258-6264.

9. Deloger M, El Karoui M, Petit MA: A genomic distance based on MUM indicates discontinuity between most bacterial species and genera. J Bacteriol 2009, 191(1):91-99.

10. Richter M, Rossello-Mora R: Shifting the genomic gold standard for the prokaryotic species definition. Proc Natl Acad Sci USA 2009, 106(45):19126-19131.

11. Didelot $X$, Maiden MC: Impact of recombination on bacterial evolution. Trends Microbiol 18(7):315-322.

12. Bergstrom CT, Lipsitch M, Levin BR: Natural selection, infectious transfer and the existence conditions for bacterial plasmids. Genetics 2000, 155(4):1505-1519.

13. Campbell AM: Lateral gene transfer in prokaryotes. Theor Popul Biol 2000, 57(2):71-77.

14. Gal-Mor O, Finlay BB: Pathogenicity islands: a molecular toolbox for bacterial virulence. Cell Microbiol 2006, 8(11):1707-1719.

15. Posada $D$, Crandall KA: The effect of recombination on the accuracy of phylogeny estimation. J Mol Evol 2002, 54(3):396-402.

16. Thomas CM, Nielsen KM: Mechanisms of, and barriers to, horizontal gene transfer between bacteria. Nat Rev Microbio/ 2005, 3(9):711-721.

17. Touzain F, Denamur E, Medigue C, Barbe V, El Karoui M, Petit MA: Small variable segments constitute a major type of diversity of bacterial genomes at the species level. Genome Biol 2010, 11(4):R45.

18. Spratt BG, Hanage WP, Feil EJ: The relative contributions of recombination and point mutation to the diversification of bacterial clones. Curr Opin Microbiol 2001, 4(5):602-606.

19. Jiggins FM: The rate of recombination in Wolbachia bacteria. Mol Biol Evol 2002, 19(9):1640-1643.

20. Jolley KA, Wilson DJ, Kriz P, McVean G, Maiden MC: The influence of mutation, recombination, population history, and selection on patterns of genetic diversity in Neisseria meningitidis. Mol Biol Evol 2005 22(3):562-569.

21. Tettelin H, Riley D, Cattuto C, Medini D: Comparative genomics: the bacterial pan-genome. Curr Opin Microbiol 2008, 11(5):472-477.

22. Souza $V$, Nguyen $\Pi$, Hudson RR, Pinero D, Lenski RE: Hierarchical analysis of linkage disequilibrium in Rhizobium populations: evidence for sex? Proc Natl Acad Sci USA 1992, 89(17):8389-8393.

23. Abby S, Daubin V: Comparative genomics and the evolution of prokaryotes. Trends Microbiol 2007, 15(3):135-141.

24. Gonzalez V, Acosta JL, Santamaria Rl, Bustos P, Fernandez JL, Hernandez Gonzalez IL, Diaz R, Flores M, Palacios R, Mora J, et al: Conserved symbiotic plasmid DNA sequences in the multireplicon pangenomic structure of Rhizobium etli. Appl Environ Microbiol 2010, 76(5):1604-1614.

25. Pinero D, Martinez E, Selander RK: Genetic diversity and relationships among isolates of Rhizobium leguminosarum biovar phaseoli. Appl Environ Microbiol 1988, 54(11):2825-2832.

26. Silva C, Le E, Souza V: Reticulated and epidemic population genetic structure of Rhizobium etli biovar phaseoli in a traditionally managed locality in Mexico. Molecular Ecology 1999, 8:277-287. 
27. Bailly X, Giuntini E, Sexton MC, Lower RP, Harrison PW, Kumar N, Young JP: Population genomics of Sinorhizobium medicae based on low-coverage sequencing of sympatric isolates. ISME J 2011.

28. Brockman W, Alvarez P, Young S, Garber M, Giannoukos G, Lee WL, Russ C, Lander ES, Nusbaum C, Jaffe DB: Quality scores and SNP detection in sequencing-by-synthesis systems. Genome Res 2008, 18(5):763-770.

29. Hubisz MJ, Lin MF, Kellis M, Siepel A: Error and error mitigation in lowcoverage genome assemblies. PLoS One 2011, 6(2):e17034.

30. Gonzalez V, Bustos P, Ramirez-Romero MA, Medrano-Soto A, Salgado H, Hernandez-Gonzalez I, Hernandez-Celis JC, Quintero V, Moreno-Hagelsieb G, Girard $L$, et al: The mosaic structure of the symbiotic plasmid of Rhizobium etli CFN42 and its relation to other symbiotic genome compartments. Genome Biol 2003, 4(6):R36.

31. Huson DH, Bryant D: Application of phylogenetic networks in evolutionary studies. Mol Biol Evol 2006, 23(2):254-267.

32. Fraser C, Hanage WP, Spratt BG: Recombination and the nature of bacterial speciation. Science 2007, 315(5811):476-480.

33. Dean CB, Nielsen JD: Generalized linear mixed models: a review and some extensions. Lifetime Data Anal 2007, 13(4):497-512.

34. Keller I, Bensasson D, Nichols RA: Transition-transversion bias is not universal: a counter example from grasshopper pseudogenes. PLoS Genet 2007, 3(2):e22.

35. Yang Z, Bielawski JP: Statistical methods for detecting molecular adaptation. Trends Ecol Evol 2000, 15(12):496-503.

36. Crossman LC, Castillo-Ramirez S, McAnnula C, Lozano L, Vernikos GS, Acosta JL, Ghazoui ZF, Hernandez-Gonzalez I, Meakin G, Walker AW, et al: A common genomic framework for a diverse assembly of plasmids in the symbiotic nitrogen fixing bacteria. PLoS One 2008, 3(7):e2567.

37. Young JP, Crossman LC, Johnston AW, Thomson NR, Ghazoui ZF, Hull KH, Wexler M, Curson AR, Todd JD, Poole PS, et al: The genome of Rhizobium leguminosarum has recognizable core and accessory components. Genome Biol 2006, 7(4):R34.

38. Segovia L, Pinero D, Palacios R, Martinez-Romero E: Genetic structure of a soil population of nonsymbiotic Rhizobium leguminosarum. App/ Environ Microbiol 1991, 57(2):426-433.

39. Rogel MA, Ormeno-Orrillo E, Martinez Romero E: Symbiovars in rhizobia reflect bacterial adaptation to legumes. Syst App/ Microbiol 2011, 34(2):96-104

40. Tun-Garrido C, Bustos P, Gonzalez V, Brom S: Conjugative transfer of p42a from rhizobium etli CFN42, which is required for mobilization of the symbiotic plasmid, is regulated by quorum sensing. J Bacteriol 2003, 185(5):1681-1692.

41. Lozano L, Hernandez-Gonzalez I, Bustos P, Santamaria RI, Souza V, Young JP, Davila G, Gonzalez V: Evolutionary dynamics of insertion sequences in relation to the evolutionary histories of the chromosome and symbiotic plasmid genes of Rhizobium etli populations. Appl Environ Microbiol 2010, 76(19):6504-6513.

42. Perez-Mendoza D, Dominguez-Ferreras A, Munoz S, Soto MJ, Olivares J, Brom S, Girard L, Herrera-Cervera JA, Sanjuan J: Identification of functional mob regions in Rhizobium etli: evidence for self-transmissibility of the symbiotic plasmid pRetCFN42d. J Bacteriol 2004, 186(17):5753-5761.

43. Silva C, Vinuesa P, Eguiarte LE, Martinez-Romero E, Souza V: Rhizobium etli and Rhizobium gallicum nodulate common bean (Phaseolus vulgaris) in a traditionally managed milpa plot in Mexico: population genetics and biogeographic implications. Appl Environ Microbiol 2003, 69(2):884-893.

44. Flores M, Morales L, Avila A, Gonzalez V, Bustos P, Garcia D, Mora Y, Guo X, Collado-Vides J, Pinero D, et al: Diversification of DNA sequences in the symbiotic genome of Rhizobium etli. J Bacteriol 2005, 187(21):7185-7192.

45. Lefebure T, Stanhope MJ: Evolution of the core and pan-genome of Streptococcus: positive selection, recombination, and genome composition. Genome Biol 2007, 8(5):R71.

46. Wu J, Yu T, Bao Q, Zhao F: Evidence of extensive homologous recombination in the core genome of rickettsia. Comp Funct Genomics 2009, 510270.

47. Retchless AC, Lawrence JG: Phylogenetic incongruence arising from fragmented speciation in enteric bacteria. Proc Natl Acad Sci USA 2010, 107(25):11453-11458

48. Wang J, Huang X: A method for finding single-nucleotide polymorphisms with allele frequencies in sequences of deep coverage. BMC Bioinformatics 2005, 6:220.
49. Gordon D, Desmarais C, Green P: Automated finishing with autofinish. Genome Res 2001, 11(4):614-625.

50. Marth GT, Korf I, Yandell MD, Yeh RT, Gu Z, Zakeri H, Stitziel NO, Hillier L, Kwok PY, Gish WR: A general approach to single-nucleotide polymorphism discovery. Nat Genet 1999, 23(4):452-456.

51. Ewing B, Green P: Base-calling of automated sequencer traces using phred. II. Error probabilities. Genome Res 1998, 8(3):186-194.

52. Lawrence $C B$, Solovyev W: Assignment of position-specific error probability to primary DNA sequence data. Nucleic Acids Res 1994 22(7):1272-1280.

53. Adams MD, Celniker SE, Holt RA, Evans CA, Gocayne JD, Amanatides PG, Scherer SE, Li PW, Hoskins RA, Galle RF, et al: The genome sequence of Drosophila melanogaster. Science 2000, 287(5461):2185-2195.

54. Darling AC, Mau B, Blattner FR, Perna NT: Mauve: multiple alignment of conserved genomic sequence with rearrangements. Genome Res 2004, 14(7):1394-1403.

55. Posada D, Crandall KA: The effect of recombination on the accuracy of phylogeny estimation. J Mol Evol 2002, 54(3):396-402.

56. Edgar RC: MUSCLE: a multiple sequence alignment method with reduced time and space complexity. BMC Bioinformatics 2004, 5:113.

57. Talavera G, Castresana J: Improvement of phylogenies after removing divergent and ambiguously aligned blocks from protein sequence alignments. Syst Biol 2007, 56(4):564-577.

58. Posada D: Evaluation of methods for detecting recombination from DNA sequences: empirical data. Mol Biol Evol 2002, 19(5):708-717.

59. Posada D, Crandall KA, Holmes EC: Recombination in evolutionary genomics. Annu Rev Genet 2002, 36:75-97.

60. Sawyer S: Statistical tests for detecting gene conversion. Mol Biol Evol 1989, 6(5):526-538.

61. Worobey M: A novel approach to detecting and measuring recombination: new insights into evolution in viruses, bacteria, and mitochondria. Mol Biol Evol 2001, 18(8):1425-1434.

62. Guindon S, Gascuel O: A simple, fast, and accurate algorithm to estimate large phylogenies by maximum likelihood. Syst Biol 2003, 52(5):696-704.

63. Yang Z: PAML 4: phylogenetic analysis by maximum likelihood. Mol Biol Evol 2007, 24(8):1586-1591.

64. Bruen TC, Philippe $H$, Bryant D: A simple and robust statistical test for detecting the presence of recombination. Genetics 2006, 172(4):2665-2681.

65. Kosakovsky Pond SL, Posada D, Gravenor MB, Woelk CH, Frost SD: GARD: a genetic algorithm for recombination detection. Bioinformatics 2006, 22(24):3096-3098.

66. Eisen MB, Spellman PT, Brown PO, Botstein D: Cluster analysis and display of genome-wide expression patterns. Proc Natl Acad Sci USA 1998, 95(25):14863-14868.

67. Posada D, Crandall KA: Selecting models of nucleotide substitution: an application to human immunodeficiency virus 1 (HIV-1). Mol Biol Evol 2001, 18(6):897-906.

68. Posada D, Crandall KA: Selecting the best-fit model of nucleotide substitution. Syst Biol 2001, 50(4):580-601.

69. Stamatakis A, Ludwig T, Meier H: RAxML-III: a fast program for maximum likelihood-based inference of large phylogenetic trees. Bioinformatics 2005, 21(4):456-463.

70. Ronquist F, Huelsenbeck JP: MrBayes 3: Bayesian phylogenetic inference under mixed models. Bioinformatics 2003, 19(12):1572-1574.

71. Shimodaira H, Hasegawa M: CONSEL: for assessing the confidence of phylogenetic tree selection. Bioinformatics 2001, 17(12):1246-1247.

72. Strimmer K, Rambaut A: Inferring confidence sets of possibly misspecified gene trees. Proc Biol Sci 2002, 269(1487):137-142.

73. Thornton K: Libsequence: a C++ class library for evolutionary genetic analysis. Bioinformatics 2003, 19(17):2325-2327.

74. Tatusov RL, Galperin MY, Natale DA, Koonin EV: The COG database: a tool for genome-scale analysis of protein functions and evolution. Nucleic Acids Res 2000, 28(1):33-36.

doi:10.1186/1471-2148-11-305

Cite this article as: Acosta et al:: Genomic lineages of Rhizobium etli revealed by the extent of nucleotide polymorphisms and low recombination. BMC Evolutionary Biology 2011 11:305. 